\title{
Caracterização das Distribuições pelas Propriedades dos Estimadores de Bayes
}

Characterization of the Distributions by the Properties of Bayes Estimators

\author{
P.C. Araújo* e J.A. Cardeal ${ }^{\dagger}$ \\ Departamento de Ciências Exatas - UEFS \\ Campus Universitário, s/n, Km 03, BR 116 \\ Feira de Santana - BA - 44031-460
}

Neste trabalho reapresentamos os resultados obtidos por Kagan e Karpov [1, 2], mostrando que: para a construção do Estimador Linear Bayseano a condição necessária e suficiente é que a priori a distribuição dos erros sejam normais.

Palavras-chave: Estimadores Lineares, Priori, Posteriori, Modelo Bayseano, Distribuição Normal

In this paper we revise the Kagan and Karpov [1, 2] work showing that: for the construction of Baysean linear estimator, the necessary and sufficient condition is that a priori the distribution of the errors are the normal distribution.

Keywords: Linear Estimators, Priori, Posteriori, Baysean Model, Normal Distribution.

\section{INTRODUÇÃO}

O método bayseano pode ser definido pelo trinômio: conhecimento, experiência e opinião $[3,4]$, e pode ser expresso na forma

$$
p(\theta / x, H)=\frac{p(\theta / H) p(x / \theta, H)}{p(x / H)},
$$

onde $\theta$ é a variável desconhecida, $H$ é a história e $x$ a variável aleatória observada [5-7]. Como

$$
p(x / H)=\int_{\Theta} p(x, \theta / H) d F(\theta, H),
$$

é uma constante, então

$$
p(\theta / x, H) \propto p(x / \theta, H) p(\theta / H) .
$$

De (1) temos que: $p(\theta / x, H)$, o conhecimento, é obtido quando temos $p(x / \theta, H)$, a experiência, e $p(\theta / H)$ sua opinião [8]. Como $H$ é comum a todos os termos podemos omiti-la e reescrever

\footnotetext{
*Endereço Eletrônico: pericles@uefs.br

${ }^{\dagger}$ Endereço Eletrônico: cardeal@uefs.br
}

o teorema de Bayes como

$$
p(\theta, x) \propto f(x / \theta) p(\theta),
$$

com $f(x / \theta)$, a verossimilhança e $p(\theta)$, a distribuição a priori do parâmetro. Portanto, o Princípio da Verossimilhança (dois resultados experimentais $x$ e $y$ possuem a mesma informação sobre $\theta$ se $f(\theta / x)$ e $f(\theta / y)$ são proporcionais [3]) é conseqüência do enfoque bayseano [9].

\section{O PARÂMETRO DE LOCAÇÃO BAYSEANO}

Seja o problema da estimação do parâmetro de locação $\theta \in R$ em $n$ observações, descritas a partir do modelo

$$
x_{i}=\theta+\varepsilon_{i} \text { para } i=1, \ldots n,
$$

$\operatorname{com} \varepsilon_{i}$ variáveis aleatórias independentes.

Assumimos que o risco do estimador $\tilde{\theta}=$ 
$\tilde{\theta}\left(x_{1}, \ldots, x_{n}\right)$, do parâmetro $\theta$, tem a forma

$$
r(\tilde{\theta})=\int_{-\infty}^{+\infty} R(\tilde{\theta}, \theta) d \Pi(\theta),
$$

com $\Pi$ a medida de probabilidade sobre $\mathbb{R}$ e $R(\tilde{\theta}, \theta)$, a função de perda. Para este caso vamos restringir a função de perda a

$$
R(\tilde{\theta}, \theta)=E_{\theta}(\tilde{\theta}-\theta)^{2} .
$$

O risco associado a $\tilde{\theta}$ é dado por (6), $\operatorname{com} \theta$ uma variável aleatória independente do vetor de erros $\left(\varepsilon_{1}, \ldots, \varepsilon_{n}\right)$ e com uma priori $\Pi$. Neste caso, o problema de estimação de $\theta$ é tratado como um problema de previsão de $\theta$, dada uma única quantidade $x_{i}$, obtida dos resultados observados.

Quando

$$
\int_{-\infty}^{\infty} \theta^{2} d \Pi(\theta)<\infty
$$

o melhor estimador de $\theta$ é a média da posteriori [1], que é a perda quadrática [10]

$$
\begin{aligned}
\hat{\theta} & =E\left(\theta / X_{1}, \ldots, X_{n}\right) \\
& =\frac{\int_{-\infty}^{+\infty} \theta \Pi_{1}^{n} F_{j}^{\prime}\left(x_{j}-\theta\right) \Pi(\theta)}{\int_{\infty}^{+\infty} \Pi_{1}^{n} F_{j}^{\prime}\left(x_{j}-\theta\right) \Pi(\theta)},
\end{aligned}
$$

com $F_{j}^{\prime}\left(x_{j}-\theta\right)$, a densidade de ditribuição $\operatorname{dos}$ erros $\varepsilon_{j}$ (independentes). Para construção do estimador (8), denominado estimador bayseano correspondente a priori de distribuição $\Pi$, é necessário o conhecimento da priori da distribuição dos erros. Uma questão natural é saber em quais circustâncias o conhecimento, apenas do primeiro e segundo momentos das distribuições $F_{j}$ e $\Pi$, é suficiente para a construção dos estimadores $\hat{\theta}=E\left(\theta / X_{1}, \ldots, X_{n}\right)$. Analiticamente, esta questão é reduzida ao estudo

$$
E\left(\theta / X_{1}, \ldots, X_{n}\right) \equiv \hat{E}\left(\theta / X_{1}, \ldots, X_{n}\right),
$$

onde $\hat{E}\left(\theta / X_{1}, \ldots, X_{n}\right)$ é simplesmente o melhor estimador linear.

Sendo $F_{j}(x)$ a função de distribuição do erro $\varepsilon_{j}$, definimos

$$
\phi_{j}(t)=\int_{-\infty}^{+\infty} e^{i t x} d F_{j}(x) .
$$

Como $\Pi(\theta)$ é a função de distribuição da priori, definimos também

$$
\varphi(t)=\int_{-\infty}^{+\infty} e^{i t \theta} d \Pi(\theta) .
$$

Os momentos de primeira e segunda ordem das funções de distribuição $F_{j}(x)$ e $\Pi(\theta)$ são dados por

$$
\begin{aligned}
\mu_{1 j} & =\int_{-\infty}^{+\infty} x d F_{j}(x), \\
\mu_{2 j} & =\int_{-\infty}^{+\infty}\left(x-\mu_{1 j}\right)^{2} d F_{j}(x), \\
\alpha_{1} & =\int_{-\infty}^{+\infty} \theta d \Pi(\theta), \\
\alpha_{2} & =\int_{-\infty}^{+\infty}\left(\theta-\alpha_{1}\right)^{2} d \Pi(\theta),
\end{aligned}
$$

onde o índice $j$ é assumido para os casos onde os erros apresentam distribuições diferentes. Consideramos, também, $\mu_{2 j}<\infty$ para $j=1, \ldots n$ e $\alpha_{2}<\infty$. Denominaremos $M_{k}(l)$ o espaço de todo polinômio em que $x_{1}, \ldots, x_{n}$ (respectivamente em $k$ ) de grau menor a 2, dotado de produto interno

$$
(Q, R)=E(Q, R)=\int E_{\theta}(Q, R) d \Pi(\theta),
$$

com

$$
\begin{aligned}
E_{\theta}(Q, R)= & \int_{R^{n}} Q\left(x_{1}, \ldots, x_{n}\right) R\left(x_{1}, \ldots, x_{n}\right) \\
& \times \prod_{1}^{n} d F_{j}\left(x_{j}-\theta\right)
\end{aligned}
$$

sendo o operador de projeção em $M_{k}$ definido por $\hat{E}\left(. / M_{k}\right)$.

\section{A. Estimador Linear Bayseano}


Quando

$$
\begin{aligned}
& 0<\alpha_{2}<\infty \\
& 0<\mu_{2 j}<\infty, \operatorname{com} j=1, \ldots n,
\end{aligned}
$$

desprezando-se os casos em que $\alpha_{2} \Pi_{1}^{n} \mu_{2 j}=0$, o melhor estimador linear de $\theta$ é o estimador bayseano

$$
\hat{E}\left(\theta / M_{1}\right)=\hat{\theta}=c_{0}+\sum_{1}^{n} c_{j} x_{j},
$$

com

$$
\begin{aligned}
c_{0} & =\frac{\alpha_{1}-\sum_{1}^{n} \frac{\alpha_{2} \mu_{1 j}}{\mu_{2 j}}}{1+\sum_{1}^{n} \frac{\alpha_{2}}{\mu_{2 j}}}, \\
c_{j} & =\frac{\frac{\alpha_{2}}{\mu_{2 j}}}{1+\sum_{1}^{n} \frac{\alpha_{2}}{\mu_{2 j}}} \text { para } j=1, \ldots, n .
\end{aligned}
$$

Kagan e Karpov (1992) afirmaram que: nos casos em que $n \geq 2$ em (14), para que tenhamos

$$
E\left(\theta / X_{1}, X_{2}, \ldots, X_{n}\right)=\hat{E}\left(\theta / X_{1}, X_{2}, \ldots, X_{n}\right),
$$

é necessário e suficiente que as distribuições $F_{j}$ $e$ П sejam normalmente distribuídas.

Para demonstrar o teorema de Karpov e Kagan, escrevemos

$$
\begin{aligned}
\theta^{\prime} & =\theta-\alpha_{1}, \\
x_{j}^{\prime} & =x_{j}-\alpha_{1}-\mu_{1 j}, j=1, \ldots, n .
\end{aligned}
$$

Assumindo que $\alpha_{1}=\mu_{1 j}=0, j=1, \ldots, n$. Então

$$
E\left(\theta / X_{1}, \ldots X_{n}\right)=\sum_{1}^{n} c_{j} x_{j} .
$$

A esperança de (18), multiplicada por $\exp i \sum_{1}^{n} t_{j} x_{j}$, é equivalente a

$$
\begin{aligned}
& E\left[\hat{\theta} \exp i \sum_{1}^{n} t_{j} X_{j}\right] \\
= & E\left[\exp i \sum_{1}^{n} t_{j} X_{j} E\left(\theta / X_{1}, \ldots X_{n}\right)\right] \\
= & E\left[\sum_{1}^{n} c_{j} X_{j} \exp i \sum_{1}^{n} t_{j} X_{j}\right] .
\end{aligned}
$$

Utilizando os resultados (10) e (11), obtemos uma equação diferencial [11] que podemos reescrever na forma

$$
\begin{aligned}
& \varphi^{\prime}(t) \prod_{1}^{n} \phi_{j}\left(t_{j}\right) \\
= & \underline{\mathrm{c}} \varphi^{\prime}(t) \prod_{1}^{n} \phi_{j}\left(t_{j}\right) \\
& +\varphi(\underline{\mathrm{t}}) \sum_{1}^{n} c_{j}\left[\phi_{j}^{\prime}\left(t_{j}\right) \prod_{k \neq j} \phi_{k}\left(t_{k}\right)\right],
\end{aligned}
$$

$\operatorname{com} \underline{\mathrm{t}}=\sum_{j=1}^{n} t_{j}$ e $\underline{\mathrm{c}}=\sum_{j=1}^{n} c_{j}$. Então, existe $\epsilon>0$ tal que, para $\left|t_{j}\right|<\epsilon$,

$$
\varphi(\underline{\mathrm{t}}) \phi\left(t_{j}\right) \neq 0 .
$$

De (19), temos

$$
(1-\underline{\mathrm{c}}) \kappa(\underline{\mathrm{t}})=\sum_{1}^{n} c_{j} h_{j}(\underline{\mathrm{t}}),
$$

$\operatorname{com} \kappa(\underline{\mathrm{t}})=\frac{\varphi^{\prime}(\underline{\mathrm{t}})}{\varphi(\underline{\mathrm{t}})}$ e $h_{j}(t)=\frac{\phi^{\prime}(\underline{\mathrm{t}})}{\phi(\underline{\mathrm{t}})}$, funções diferenciáveis, para $|t|$ suficientemente pequeno (14). De $(16), c_{j} \neq 0$ e $(1-\underline{c}) \neq 0$. Derivando (21) em relação a $t_{j}$ e tomando $t_{j}=0$, obtemos $\kappa^{\prime}(\mathrm{t})=$ constante em alguma vizinhança de zero. Por hipótese, $\varphi^{\prime}(0)=\phi^{\prime}(0)=0$, portanto

$$
\begin{aligned}
\varphi^{\prime}(t) & =\exp \left(\lambda_{j} t^{2}\right) \mathrm{e} \\
\phi_{j}^{\prime}(t)= & \exp \left(\lambda_{j} t^{2}\right) \text { para } \\
\mid & t \mid<\varepsilon^{\prime}
\end{aligned}
$$

provando assim que $F_{j}$ e $\Pi$ têm distribuição normal (condição necessária). A suficiência segue de: se o vetor $\left(\theta, X_{1}, \ldots, X_{n}\right)$ é normalmente distribuído, então o problema do melhor preditor para uma componente do vetor tem solução linear [12]. Portanto o teorema está provado.

\section{AGRADECIMENTOS}

Os autores agradecem aos árbitros deste trabalho e ao Prof. Franz Farias pela infinita paciência. 
[1] A.M. Kagan, Y.N. Karpov, Bayesian formulation of the estimation problem for the location parameter. Leningrad Section of the Mathematical Institute. Zap. Nauch. Semi 29, (1972).

[2] A.M. Kagan, C.R. Rao, Characterization Problems in Mathematical Statistic. Canada: Wiley International (1973).

[3] C.A.B. Pereira, M.A. Viana, Elementos de Inferência Bayeseana. In: Simpósio Nacional de Probabilidade e Estatística, 5º 1985. Resumos. São Paulo: ABE (1985).

[4] C.A.B Pereira, Estatística e Informação. Bol. C. L. da ABE Ano XI, (1995).

[5] D. Gamerman, H.S. Migon, Inferência Estatística: Uma Abordagem Integrada. Rio de Janeiro: IM-UFRJ (1993).

[6] P.C. Araújo, Método Linear Bayseano. Dissertação (Mestrado) - Instituto de Matemática e Estatística da USP, São Paulo (1997).

[7] P.C. Araújo, S. Wechesler, Método Linear
Bayeseano. In: Simpósio Nacional de Probabilidade e Estatística, 22º ${ }^{\circ}$ 1996. Resumos. Caxambu: ABE (1996).

[8] R.H. Loschi, Coerência, Probabilidade e Calibração. Dissertação (Mestrado) - Instituto de Matemática e Estatística da USP, São Paulo (1992).

[9] L.Y.T. Inoue, Desenvolvimento e Implicações do Princípio da Verossimilhança. Dissertação (Mestrado) - Instituto de Matemática e Estatística da USP, São Paulo (1995).

[10] H. Bolfarine, M.C. Sandoval, Introdução à Inferência Estatística. Rio de Janeiro: SBM (2001).

[11] C.P. Simon, L. Blume, Mathematics for Economists. New York: W.W. Norton \& Company (1994).

[12] M. Kendall, A. Stuart, The Advanced Theory of Statistics. vol. 2. London: Hafner Publishing Co. (1979). 